\title{
Middle Turbinate Evacuation Conchoplasty in Management of Contact-Point Rhinogenic Headache in Children
}

\author{
Osama A. Albirmawy ${ }^{\mathrm{a}, \mathrm{b}}$, Hossam S. Elsherif ${ }^{\mathrm{a}}$, Emad M. Shehata ${ }^{\mathrm{a}}$, Ahmed Younes $^{\mathrm{a}}$
}

\begin{abstract}
Background: Our objective is to evaluate the efficacy and outcomes of endoscopic evacuation of middle turbinate "concha bullosa" compared with lateral partial turbinectomy, in children with chronic contact-point headaches. For the research, this study is using prospective clinical trial and setting in Otolaryngology department, Tanta University Hospital, Egypt.
\end{abstract}

Methods: Over three years, 60 children underwent surgery for management of contact-point rhinogenic chronic headache resulting from middle turbinate concha bullosa, using either an evacuation technique ( 30 children) or lateral partial turbinectomy (lamellectomy technique, 30 children) with at least 12 months' follow up. Post-operative adhesions, olfactory disorders, pain intensity, and frequency and duration of headache attacks were monitored.

Results: None of the children of the evacuation group developed post-operative synechia or olfactory disorders. In the lamellectomy group, two children reported reduced olfactory capacity and an additional four children had developed adhesions. Pain intensity and frequency and duration of headache attacks improved significantly in both groups (pre- vs post-operative results), but significantly more so in the evacuation group.

Conclusions: The evacuation technique may be superior to the lamellectomy technique in preventing post-operative synechia and olfactory disorders, as well as better relieving of pain intensity and frequency and duration of headache attacks.

Keywords: Concha bullosa; Contact-points; Headache; Cephalgia

Manuscript accepted for publication October 2, 2012

${ }^{a}$ Otolaryngology Department, Tanta University Hospital, Egypt

${ }^{\mathrm{b}}$ Corresponding author: Osama A. Albirmawy, Otolaryngology

Department, Tanta University Hospital, Reyad St., 88, Tanta, 31211,

Gharbeya, Egypt. Email: albirmawy@hotmail.com

doi: http://dx.doi.org/10.4021/ijcp53w

\section{Introduction}

Chronic headache in children is not uncommon, and the etiologic factors are quite varied [1]. Rhinogenic related headache has been recognized since 1888 [2]. However, not much attention has been given to it until the advent of nasal endoscopy and the use of computerized tomography (CT) scanning [3].

Concha bullosa, which is the pneumatization of the concha, is commonly encountered in the middle turbinate and rarely found in the superior and inferior conchae [4-8]. Mucosal contact between the concha bullosa and the nasal septum or the lateral nasal wall has resulted in the release of substance P [9], Calcitonin gene-related peptide (CGRP) [10], and neurokinin A [11]. These chemical mediators are well-recognized in nocioceptive fibers in the central nervous system and the trigeminovascular system [9-11]. Therefore, contact points may be a cause of secondary headache or a trigger factor for primary headaches [12].

Contact-points cephalgia is a newly added headache disorder in the International Classification of Headache Disorders (ICHD-2) [13], supported by limited evidence. It is characterized by; intermittent pain localized in the periorbital and medial canthal or temporozygomatic regions; evidence of mucosal contact points by nasal endoscopy or CT scanning; gravitational variation of pain with postural movements; abolition of headache within 5 minutes following topical application of local anesthesia to the contact point area; and significant resolution of the headache in less than 7 days after removal of mucosal contact points.

Some investigators have described their experience treating trigeminovascular system-related headaches using transection of the 5th cranial nerve or injecting the Gasserian ganglion, using alcohol or novacaine [14-16]. Prior to endoscopic sinus surgery, total middle turbinectomy was used to manage concha bullosa [17]. With the evolution of endoscopic sinonasal surgery, investigators have described different techniques of partial turbinectomy and turbinoplasty aiming to relief contact-point headaches and reduce postoperative synechia [8, 18-24].

This study aimed to evaluate the efficacy and outcomes 

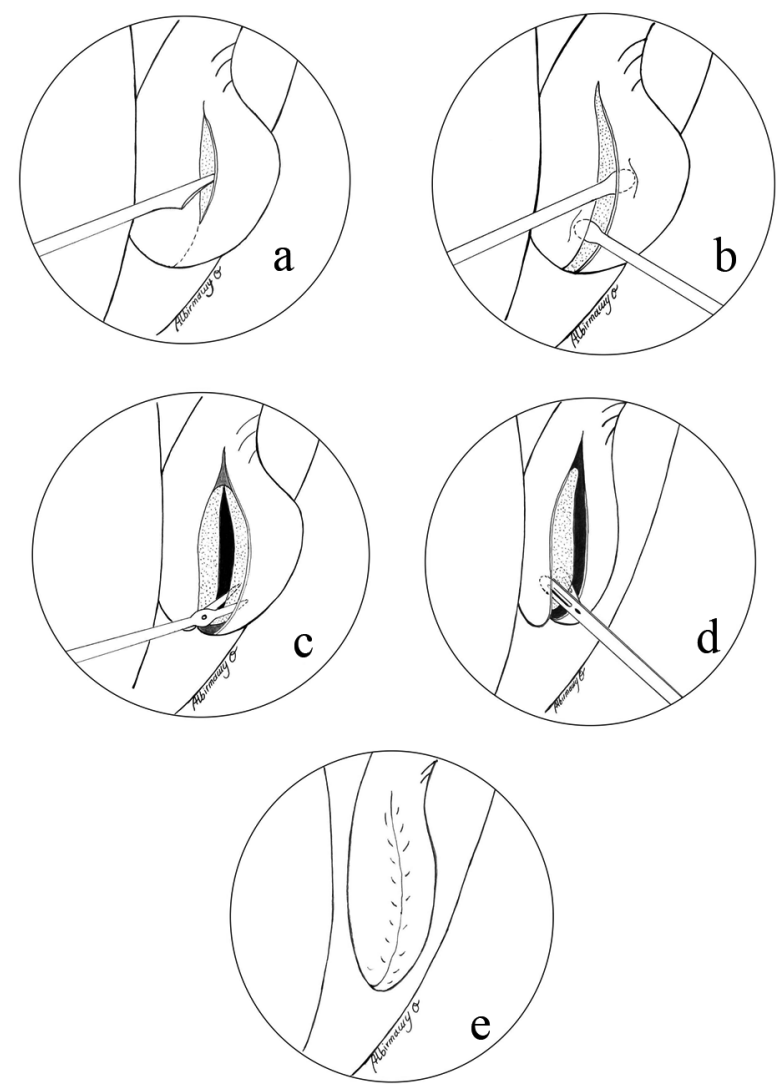

Figure 1. (a) Antero-inferior vertical mucosal incision using sickle knife; (b) Raising the medial and lateral mucoperiosteal flaps; (c) Dividing the bony concha into its lateral and medial laminae; (d) Extracting the medial and lateral bony laminae; (e) The final appearance of the debulked concha.

of endoscopic evacuation of middle turbinate concha bullosa, as a novel technique, compared with lateral partial turbinectomy $[19,25]$, in a selected group of children with chronic headaches not responding to medical treatment.

\section{Patients and Methods}

Patient population and selection criteria

Over three years, 60 children were selected for surgery in this double-arm prospective randomized parallel study, based on the following criteria:

Headache: All children were suffering from frequent attacks of long-lasting headache, refractory to medical treatment for at least one year, as diagnosed by two neurologists [13]. Sinusitis and nasal allergy were excluded by clinical examination and CT scans. Other systemic causes of headache such as anemia and neurovascular disorders were also excluded. There was no associated aura or autonomic hyperactivity as observed in migraine or in cluster headache as well as no response to medications used for vascular headache.
Contact point: Both preoperative endoscopic examination and CT scans had to demonstrate a mucosal contact between the middle turbinate "concha bullosa", as defined by Bolger et al [6], and the nasal septum or bulla ethmoidalis on the predominant side of the headache. Any other contact points between the nasal septum and the lateral nasal wall rather than the middle turbinate were excluded.

Topical anesthetic test: All children were asked to visit the clinic during an acute attack of headache. A cotton swab containing normal saline solution was applied to the suspected endonasal contact area for 30 seconds. The reaction of the child was considered false positive if headache was relieved or the severity was reduced, therefore, the child was excluded. If the headache persisted, the cotton swab was replaced by cotton pledget containing $10 \%$ Lidocaine $\mathrm{HCl}$. The test was considered positive if the persisting pain was immediately relieved or the severity was reduced by $50 \%$ or more after 5 minutes, compared with the visual analogue scale measuring pain severity before the test.

Preoperatively, based on the medical history of the selected cases, the average number of headache attacks per month and the mean duration of each attack were calculated. The pain intensity was also reported and measured on a vi- 
Table 1. Patent Profiles.

\begin{tabular}{|c|c|c|}
\hline Parameter & Evacuation group* & Lamellectomy group* \\
\hline \multicolumn{3}{|l|}{ Age (years) } \\
\hline Range & $9-16$ & $10-15$ \\
\hline Mean & 13 & 12 \\
\hline SD & 1.8 & 2.1 \\
\hline \multicolumn{3}{|l|}{$\operatorname{Sex}(n)$} \\
\hline Boys & 18 & 16 \\
\hline Girls & 12 & 14 \\
\hline \multicolumn{3}{|c|}{ Surgical procedures (n) } \\
\hline Total & 40 & 39 \\
\hline Bilat & 10 & 9 \\
\hline \multicolumn{3}{|c|}{ Duration of surgery (min) } \\
\hline \multicolumn{3}{|c|}{ Bilat concha } \\
\hline Range & $60-110$ & $55-100$ \\
\hline Mean & 70 & 65 \\
\hline SD & 9 & 4 \\
\hline \multicolumn{3}{|c|}{ Unilat concha } \\
\hline Range & $45-95$ & $40-95$ \\
\hline Mean & 55 & 52 \\
\hline SD & 7 & 8 \\
\hline \multicolumn{3}{|c|}{ Chronicity period (months) } \\
\hline Range & $31-43$ & $41-48$ \\
\hline Mean & 19 & 21 \\
\hline SD & 6 & 7.5 \\
\hline
\end{tabular}

${ }^{*} \mathrm{n}=30$ cases; $\mathrm{SD}=$ standard deviation; $\mathrm{n}=$ number; Bilat = bilateral; Unilat = unilateral; min = minutes

sual analogue scale ranging from 0 to 100 . An informed consent was provided by the parents who were not aware of the surgical technique. The Ethics Committee of the University of Tanta approved the study.

\section{Surgical technique}

The children were randomly operated under general anesthesia by the same surgeon and divided into two equal groups of 30 children each. The concha bullosae of the control group were managed using endoscopic partial lateral turbinectomy (lamellectomy group) as described by Meserklinger [19] and Kennedy and Zinreich [25]. The study group comprised those whose concha bullosae were managed by endoscopic evacuation conchoplasty (evacuation group) using the following steps:

Step 1: Routine preparation. Under general anesthesia, cotton wool pledgets soaked in 1:1000 adrenaline solution were placed in the middle meatus and nasal cavity and left for 10 minutes. Using endoscopic guidance, $0.5 \mathrm{~mL}$ of 2 percent lignocaine/adrenaline was injected submucosaly into the anterior/inferior surface of the concha bullosa. 
(a)

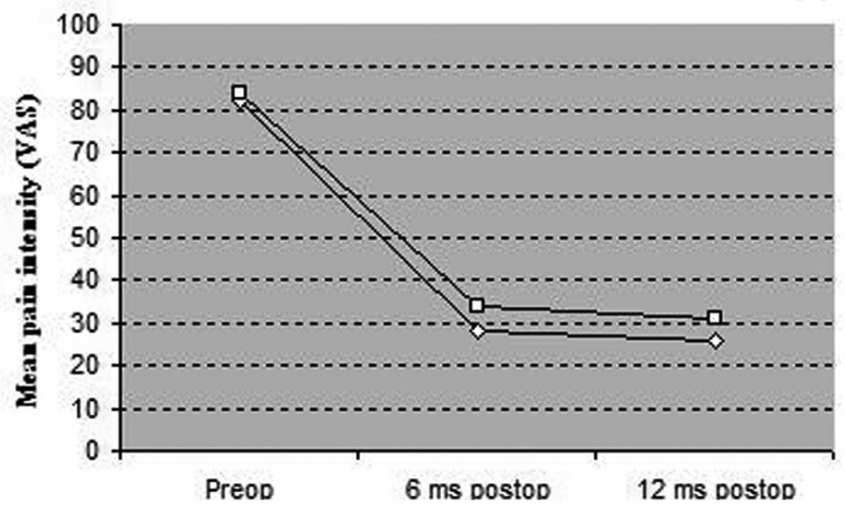

(b)

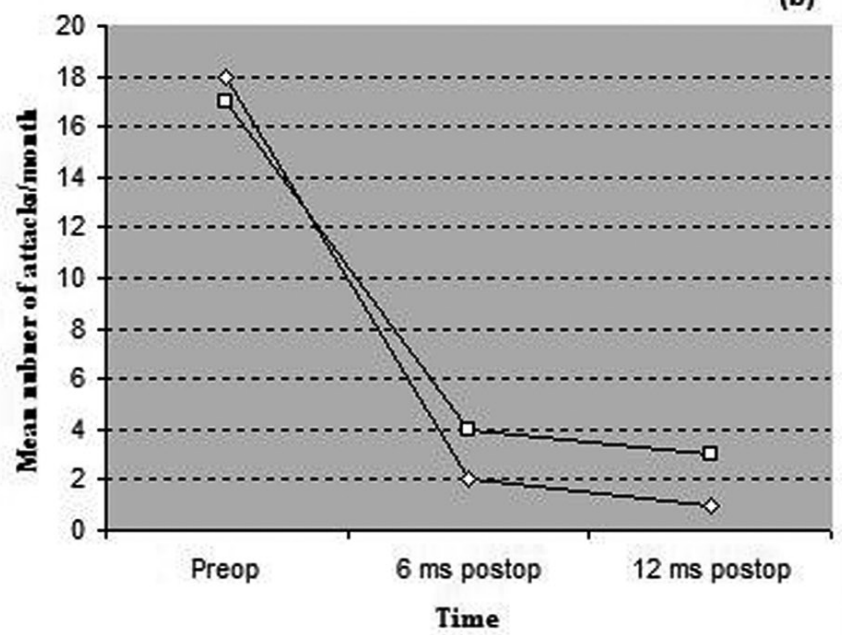

(c)

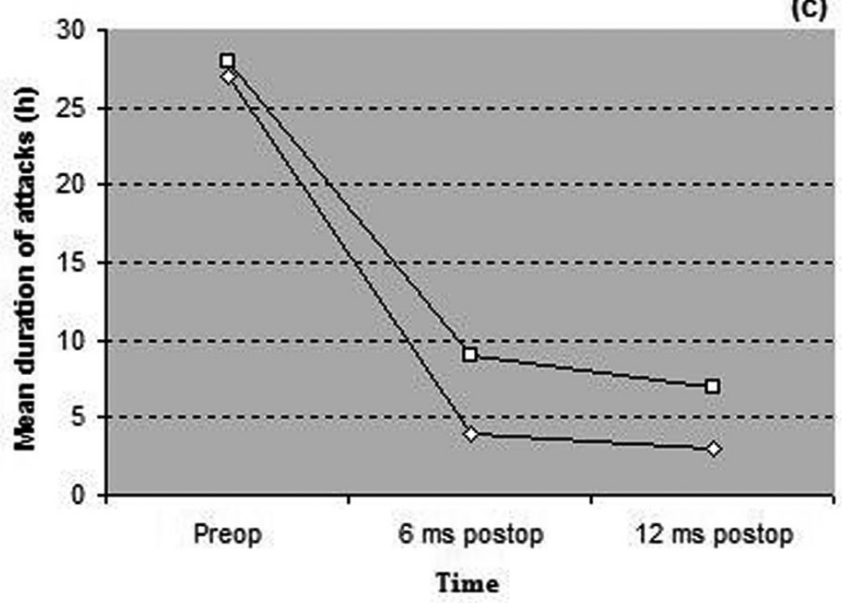

$\neg$ Evacuation group $\rightarrow-$ Lamellectomy group

Figure 2. Progressive results for (a) mean pain intensity, (b) mean numbers of headache attacks/ month and (c) mean duration of attacks, in the surgical groups

Step 2: Creating mucoperiosteal flaps. A sickle knife was used to make a vertical incision into the anterior face of the concha bullosa and extended along its inferior surface (Fig. 1a). A dissector was carefully used to create a plane be- tween the bony walls of the concha and its covering medial and lateral mucoperiosteal surfaces (Fig. 1b). This created superiorly and posteriorly based medial and lateral mucosal flaps. 
Step 3: Evacuation of the bony concha. A straight scissors was used to divide the bony concha into lateral and medial lamellae near its anterior and inferior borders (Fig. 1c). This sharp division along with preservation of the ground lamella and the vertical lamella prevented destabilization of the middle turbinate. The covering mucosal flaps were then sequentially raised until the bony lateral and medial lamellae could be removed (evacuated) using Blakesley forceps (Fig. 1d).

Step 4: Flap repositioning. Both medial and lateral mucoperiosteal flaps were then laid over their raw surfaces closing the evacuated conchal cavity (Fig. 1e). Gelfilm was placed in the middle meatus and the nasal cavity to help hold flaps in place.

\section{Postoperative care and outcome measures}

Prophylactic oral antibiotic was prescribed for a few days after surgery. Children were started on normal saline spray from the first post-operative day. Follow-up was scheduled every 2 weeks for 1 month and every month for at least one year. These visits included an endoscopic examination of the nose under local anesthesia to detect any adhesions or the need for toileting of the middle meatus. A detailed history including questions about frequency of occurrence and duration of attacks, and analogue scaling of pain intensity was documented. Any post-operative olfactory disorder was also reported.

\section{Statistical analysis}

To avoid bias by the clinical team, the coauthors who were not involved in the clinical and surgical treatment of the cases had followed the outcome of subjective symptoms including pain severity and frequency and duration of headache attacks at 6 and 12 post-operative months, and compared them with the preoperative findings. Changes were rated using a MANOVA, and a significance level of .05 was assumed. Children with a complete cessation of symptoms were considered "cured"; children with a significant decrease in symptoms were considered "improved"; lack of significant change was considered "unchanged". Comparisons of the three categories between the preoperative results and the results obtained at 6 and 12 months postoperatively were carried out within and in-between the surgical groups using a $t$-test.

\section{Results}

The surgery was tolerated well by all children. Neither exacerbation of pain intensity nor an increase in the frequency or duration of attacks after surgery was observed in any of the surgical groups. When used as factors in the ANOVA, neither age nor gender had any significant effect on pain intensity or on duration and frequency of attacks. The mean follow-up period was 18 months with a minimum of 12 months. Table 1 shows the patient profiles in both groups. The mean chronicity period of headaches before surgical intervention was 19 months (range, 13 - 43 months) in the evacuation group, and 21 months (range, 14 - 48 months) in the lamellectomy group $(\mathrm{p}>0.05)$.

Postoperatively, two children in the lamellectomy group reported reduced olfactory capacity that persisted; an additional four children had developed adhesions between the middle turbinate and the lateral nasal wall. Endonasal revision surgery for these children, under local anesthesia, successfully resolved the problem. None of the children of the evacuation group developed postoperative synechia or olfactory disorders. Although none of the patients consented to postoperative $\mathrm{CT}$ scans in order to show the relief of contact points, the postoperative endonasal evaluation showed patent nasal fossae with absence of contact points in all cases.

In the evacuation group, pain intensity decreased significantly from a preoperative level of $82( \pm 6)$ to $28( \pm 5)$ at 6 -months postoperatively $(\mathrm{p}=0.001)$ and decreased slightly, but not significantly, to $26( \pm 7)$ at the 12-month. Likewise, in the lamellectomy group, pain intensity decreased significantly from $84( \pm 2)$ preoperatively to $34( \pm 8)$ at the 6 -month postoperatively $(\mathrm{p}=0.021)$ and further decreased, but not significantly, to $31( \pm 4)$ at the end of the first year (Fig. 2a). There was no statistically significant difference in the results of the preoperative pain intensity on comparing the surgical groups. However, the difference in the follow-up results was statistically significant $(\mathrm{p}=0.03)$, both at 6 - and 12 -months postoperatively.

The frequency of the attacks per month decreased from a mean preoperative level of $18( \pm 3)$ in the evacuation group and $17( \pm 5)$ in the lamellectomy group to $2( \pm 2)$ in the evacuation group and $4( \pm 3)$ in the lamellectomy group at 6 -months postoperatively $(\mathrm{p}=0.001)$ and decreased again, but not significantly, to $1( \pm 1)$ in the evacuation group and $3( \pm 2)$ in the lamellectomy group at the 12-month (Fig. 2b). Although the preoperative level was almost equivalent in both groups, the decrease in the frequency of attacks in the evacuation group, compared to the lamellectomy group, was statistically significant at 6 and 12 months postoperatively $(p=0.03)$. Changes in duration of the attacks showed similar findings. The mean duration decreased significantly from 27 hours $( \pm 8)$ in the evacuation group and 28 hours $( \pm 7)$ in the lamellectomy group preoperatively to 4 hours ( \pm $6)$ in the evacuation group and 9 hours $( \pm 8)$ in the lamellectomy group at 6 months postoperatively and to 3 hours $( \pm 2)$ in the evacuation group and 7 hours $( \pm 3)$ in the lamellectomy group at 12 months postoperatively, as depicted in Figure 2c.

At 6 months postoperatively, 17 children of the evacuation group were completely free of pain; 8 children had sig- 
(a)

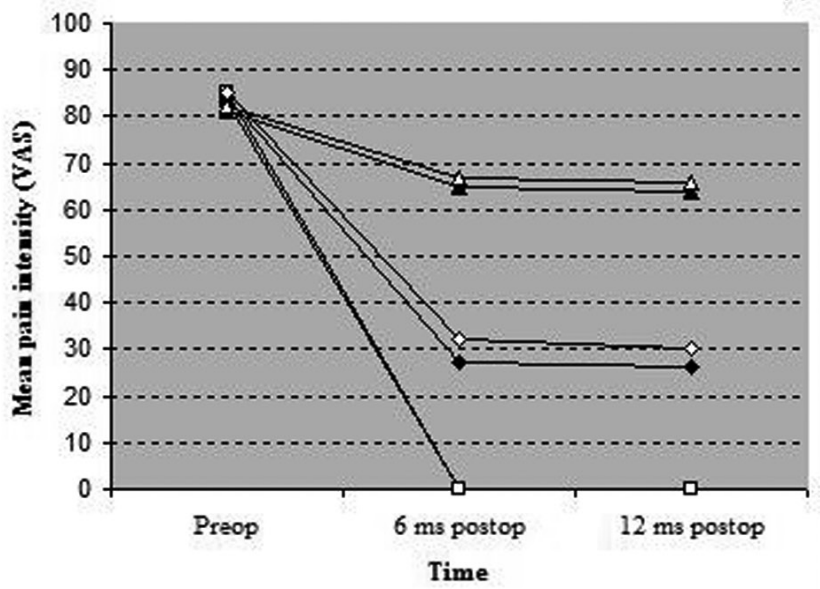

(b)

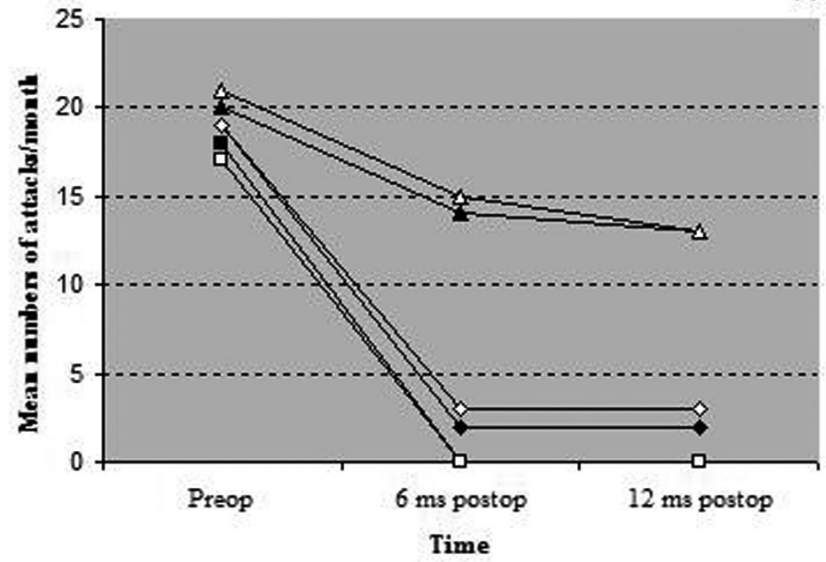

(c)

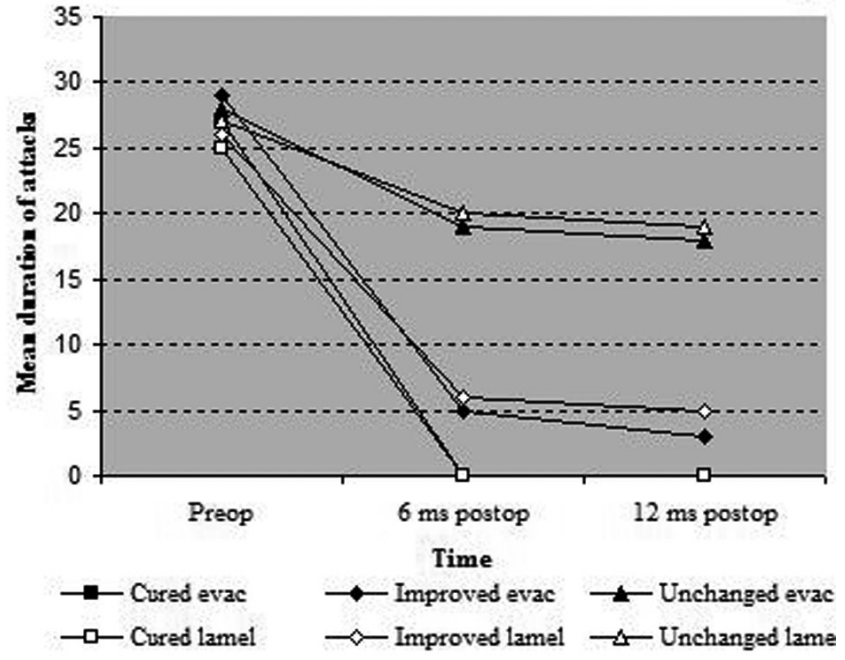

Figure 3. Progressive results for (a) mean pain intensity, (b) mean numbers of headache attacks/ month and (c) mean duration of attacks, in the categories of cured, improved and unchanged patients in each surgical group. 
nificantly improved; and 5 were unchanged. At the end of the first year, 16 children remained completely free of pain; 10 were significantly improved; and 4 had the same level of complaints as before surgery. In the category of improved but not completely cured children, pain intensity decreased from a preoperative level of $84( \pm 8)$ to $27( \pm 4)(p=0.012)$; frequency of attacks from $19( \pm 9)$ per month preoperatively to $2( \pm 1)$ per month $(\mathrm{p}=0.014)$; and duration of attacks from $29( \pm 6)$ hours to $5( \pm 3)$ hours $(\mathrm{p}=0.01)$.

In the lamellectomy group, 15 children had improved completely; 7 children were significantly improved; and 8 were unchanged at the 6-month postoperatively. After 12 months postoperatively, pain score of the children, who were completely cured, did not change; 9 children had significantly improved; and 6 had the same preoperative pain score. The pain intensity of the category of children who were improved decreased from a preoperative level of $85( \pm 3)$ to $32( \pm 2)(\mathrm{p}=0.01)$; frequency of attacks from $19( \pm 4)$ per month preoperatively to $3( \pm 2)$ per month $(\mathrm{p}=0.02)$; and duration of attacks from $26( \pm 5)$ hours to $6( \pm 3)$ hours $(\mathrm{p}=$ 0.01 ). Figure 3 depicts the differences among the three categories within and in-between the surgical groups at 6 and 12 months postoperatively, in comparison with the preoperative findings.

\section{Discussion}

Middle turbinate 'concha bullosa' is a common anatomical variant that may contribute to irritating contact-point headaches, even in children [1]. Endoscopic lateral lamellectomy is the current gold standard for treatment $[19,25]$. However, recurrence of contact points and postoperative adhesions with subsequent frontal sinus disease are common complications of this technique $[24,26]$. In the current series, the evacuation technique is a simple modification in which the bony skeleton of the concha bullosa is submucosaly evacuated leaving the anterior sagital segment of the middle turbinate debulked with smooth mucosal surfaces not in contact with the nasal septum or the lateral nasal wall. This makes the technique more conservative and able to reduce postoperative adhesions and olfactory disorders. This result was concomitant with that of Sigston et al [22] who tried a modification to partial lateral turbinectomy using the lateral posteriorly pedicled mucosal flap of the concha, after extracting its bony lamina, to cover the raw surface of the medial lamella. They hypothesized that maintaining the medial and lateral mucosa with its secretory elements decreases the risk of atrophic rhinitis and postoperative adhesions, and avoids olfactory disorders while speeding recovery from contact point headaches. However, small diseased rests of the mucosa lining the remaining medial lamella may result in the formation of mucoceles [22]. In the evacuation group of the current study, in order to avoid rests of mucosa and subse- quent mucoceles, both bony laminae of the concha together with their lining mucosa were completely extracted without adding a statistically significant extra time to the procedure.

The rhinogenic headache has been well known since 1888 [2] and the central role of the sphenopalatine ganglion in facial pain has been reported by Sluder since 1927 [27]. The middle turbinate headache syndrome was first described by Morgenstein and Krieger [28] in 1980 and the pathophysiology has been discussed in detail by Stammberger and Wolf [9] as well as by Clerico [29]. However, to the authors' best knowledge, there is little published information discussing contact points rhinogenic headache in the pediatric population. Thus, clinical researchers face challenging questions regarding the inclusion criteria, the best surgical technique and the postoperative follow-up measures in children. These children usually would have exhausted the medical profession and eventually some of them will end up in an otolaryngology office. While performing studies measuring the distance from the anterior nasal spine to the natural ostium of the sphenoid, Smith et al [30] have moved the middle and superior turbinates laterally. Unintentionally, this relieved many of the contact-point headaches in their pediatric population. Since that time, endoscopic management of concha bullosa, using different techniques, has been able to offer some relief to these children with variable rates of success $[1,28,31]$. The selection of children might benefit from a surgical procedure is critical and might explain why the reported rate from surgery is only 30 to 70 percent $[1,32]$. In the current study, the authors excluded those children who demonstrated contact points between the nasal septum and any part of the lateral nasal wall rather than the conchal part of the middle turbinate, on CT scan, to ensure that contact between the middle turbinate and the septum or the lateral nasal wall was solely the cause of the headaches. This was reaffirmed by an endoscopic nasal examination and positive topical anesthetic test. Performing a "topical anesthesia test" before surgery thereby enabled the authors to select children who reported an improvement. Nevertheless, this test did not guarantee the selection of those patients who would benefit from surgery, as reported by different authors [31-33]. However, none of these authors has found a distinct correlation between a positive test result and a surgical outcome. In addition, a placebo effect of the test cannot be excluded. Therefore, it is assumed that this test is only of limited value in helping to select patients. However, an incorrect surgical procedure that did not identify and remove the involved contact area cannot be excluded. Other factors, such as central modulation of pain may also be important [34].

In this study, postoperative pain intensity and frequency and duration of attacks were fully investigated. In the evacuation group, 6 months postoperatively, 25 of 30 children $(83 \%)$ were either free of pain or experienced significant relief in pain intensity and frequency and duration of attacks. At the end of the first year postoperatively, only 4 children 
(13\%) had the same level of complaints as before surgery. The children of the lemellectomy group had experienced a success rate of $73 \%, 6$ months postoperatively. This rate increased to $80 \%$, almost 12 months postoperatively. Although pain intensity and frequency and duration of attacks had significantly improved after surgery in both arms of the study, the success rate was significantly higher in the evacuation group than of the lamellectomy control group. This can be explained by the ability of the evacuation technique to adequately reduce the size of the concha bullosa preventing the recurrence of contact points and postoperative adhesions. The increase in the success rate, in the evacuation group, from $83 \%$ after 6 months to $87 \%$ after 12 months compared with the control group (from $73 \%$ to $80 \%$ ) may underscore the importance of the evacuation technique as being effective in allowing more shrinkage of the conchal mucosal tissue with time. The endoscopic follow-up examination suggested synechiae a cause for persisting headache in children of the lamellectomy group who did not experience significant recovery. However, postoperative CT scan, which unfortunately was not scheduled in this study, is the only investigation required to confirm this suggestion.

The clear connection between the contact points and the assumption that they are the primary cause for the headache cannot be unequivocally proven to date [35], at least in children. However, it is important for otolaryngologists to bear in mind the cause of headaches attributable to rhinogenic contact points. The data obtained in this study demonstrated that the timely recognition of contact points as well as proper surgical intervention may make a significant difference in the lives of these children. Furthermore, an adequate followup period of at least one year and the presence of a control group may act as strength factors. In 1992, Novak and Makek [36] reported a $78.5 \%$ cure rate in a group of 299 patients; their study lacked a control group as well as definitions of a follow-up period or of improvement, and no statistical analysis was undertaken. There have been various case reports of successful outcomes and short follow-up periods [37, 38]. In 1997, Clerico et al [31] have reported results from 19 patients treated surgically for refractory primary headaches during a mean follow-up period of 21 months and the success rate was $79 \%$. However, success rates of almost $90 \%$ have been reported for shorter follow-up periods of about 13 months [1, 32, 39], while after a follow-up period of 2 years, Welge-Luessen et al [34] showed an $85 \%$ success rate among their patients of which $60 \%$ were completely free of pain and $25 \%$ experienced a significant improvement. This rate decreased to $65 \%$, with only six patients being free of symptoms after 10 years.

\section{Conclusions}

Based on the results of this study, it was clear that children with headaches refractory to conservative therapy should be seen not only by a neurologist but also by an otolaryngologist, who should carefully examine the nose endoscopically, searching for possible contact points. A CT scan of the paranasal sinuses and topical anesthetic test should be performed additionally as part of the evaluation. Postoperative objective and subjective findings clarified that surgical evacuation of the skeleton of the middle turbinate "concha bullosa" was superior to the lateral lamellectomy technique with respect to the significant relief of pain intensity and frequency and duration of attacks as well as prevention of postoperative synechia and olfactory disorders. However, conducting studies on a higher number of children and having longer follow-up periods can focus more light on this issue to obtain more precise data and open a way to eliminate or ameliorate children suffering.

\section{References}

1. Parsons DS, Batra PS. Functional endoscopic sinus surgical outcomes for contact point headaches. Laryngoscope. 1998;108(5):696-702.

2. Roe JO. The frequent dependence of persisted and so called congestive headaches upon abnormal conditions of the nasal passages. Med Record 1888;34:200-4.

3. Kunachak S. Middle turbinate lateralization: a simple treatment for rhinologic headache. Laryngoscope. 2002;112(5):870-872.

4. Mohebbi A, Memari F, Mohebbi S. Endonasal endoscopic management of contact point headache and diagnostic criteria. Headache. 2010;50(2):242-248.

5. Hatipoglu HG, Cetin MA, Yuksel E. Concha bullosa types: their relationship with sinusitis, ostiomeatal and frontal recess disease. Diagn Interv Radiol. 2005;11(3):145-149.

6. Bolger WE, Butzin CA, Parsons DS. Paranasal sinus bony anatomic variations and mucosal abnormalities: CT analysis for endoscopic sinus surgery. Laryngoscope. 1991;101(1 Pt 1):56-64.

7. Kayalioglu G, Oyar O, Govsa F. Nasal cavity and paranasal sinus bony variations: a computed tomographic study. Rhinology. 2000;38(3):108-113.

8. Behin F, Behin B, Bigal ME, Lipton RB. Surgical treatment of patients with refractory migraine headaches and intranasal contact points. Cephalalgia. 2005;25(6):439443.

9. Stammberger H, Wolf G. Headaches and sinus disease: the endoscopic approach. Ann Otol Rhinol Laryngol Suppl. 1988;134:3-23.

10. Durham PL. Calcitonin gene-related peptide (CGRP) and migraine. Headache. 2006;46 Suppl 1:S3-8.

11. Goadsby PJ, Hoskin KL, Storer RJ, Edvinsson L, Connor HE. Adenosine A1 receptor agonists inhibit trigeminovascular nociceptive transmission. Brain. 2002;125(Pt 
6):1392-1401.

12. Behin F, Lipton RB, Bigal M. Migraine and intranasal contact point headache: is there any connection? Curr Pain Headache Rep. 2006;10(4):312-315.

13. Headache Classification Committee of the International Headache Society. Classification and diagnostic criteria for headache disorders, cranial neuralgias, and facial pain, 2nd edition. Cephalgia 2004;24(S1):1-160.

14. Rowbotham GF. Pain pathway in migraine. Br Med J 1942;2:685-7.

15. Harris W. Three Cases of Alcohol Injection of the Gasserian Ganglion for Trigeminal Neuralgia. Proc R Soc Med. 1912;5(Clin Sect):115-119.

16. Penfield W. Operative treatment of migraine and observations on the mechanism of vascular pain. Trans Am Acad Ophthalmol Otolaryngol 1932;37:50-64.

17. Pochon N, Lacroix JS. Incidence and surgery of concha bullosa in chronic rhinosinusitis. Rhinology. 1994;32(1):11-14.

18. Pirsig W. Reduction of the middle turbinate. Rhinology 1972;10:103-8.

19. Messerklinger W. Endoscopy of the Nose. Baltimore: Urban \& Schwarzenberg, 1978:50.

20. May M, Levine HL, Mester SJ, Porta N. Endoscopic sinus surgery. In: Levine HL, May M, (eds). Endoscopic Sinus Surgery. New York: Thieme Medical Publishers, 1993:105-75.

21. Wigand ME, Hosemann WG. Results of endoscopic surgery of the paranasal sinuses and anterior skull base. J Otolaryngol. 1991;20(6):385-390.

22. Sigston EA, Iseli CE, Iseli TA. Concha bullosa: reducing middle meatal adhesions by preserving the lateral mucosa as a posterior pedicle flap. J Laryngol Otol. 2004;118(10):799-803.

23. Woolford TJ, Jones NS. A concha bullosa crusher for use in endoscopic sinus surgery. J Laryngol Otol. 2000;114(3):205-206.

24. Har-el G, Slavit DH. Turbinoplasty for concha bullosa: a non-synechiae-forming alternative to middle turbinectomy. Rhinology. 1996;34(1):54-56.

25. Kennedy DW, Zinreich SJ. The functional endoscopic approach to inflammatory sinus disease: Current perspectives and technique modifications. Am J Rhinol 1988;2:89-96.
26. Swanson P, Lanza DC, Kennedy DW, Vining EM. The effect of middle turbinate resection upon frontal sinus disease. Am J Rhinol 1995;9:191-5.

27. Sluder G. Nasal Neurology, Headaches, and Eye Disorders. St Louis: C.V. Mosby, 1927:31-67.

28. Morgenstein KM, Krieger MK. Experiences in middle turbinectomy. Laryngoscope. 1980;90(10 Pt 1):15961603.

29. Clerico DM. Pneumatized superior turbinate as a cause of referred migraine headache. Laryngoscope. 1996;106(7):874-879.

30. Smith WC, Boyd EM, Parsons DS. Pediatric sphenoidotomy. Otolaryngol Clin North Am. 1996;29(1):159167.

31. Clerico DM, Evan K, Montgomery L, Lanza DC, Grabo D. Endoscopic sinonasal surgery in the management of primary headaches. Rhinology. 1997;35(3):98-102.

32. Tosun F, Gerek M, Ozkaptan Y. Nasal surgery for contact point headaches. Headache. 2000;40(3):237-240.

33. Mokbel KM, Abd Elfattah AM, Kamal el S. Nasal mucosal contact points with facial pain and/or headache: lidocaine can predict the result of localized endoscopic resection. Eur Arch Otorhinolaryngol. 2010;267(10):1569-1572.

34. Welge-Luessen A, Hauser R, Schmid N, Kappos L, Probst R. Endonasal surgery for contact point headaches: a 10-year longitudinal study. Laryngoscope. 2003;113(12):2151-2156.

35. Abu-Bakra M, Jones NS. Prevalence of nasal mucosal contact points in patients with facial pain compared with patients without facial pain. J Laryngol Otol. 2001;115(8):629-632.

36. Novak VJ, Makek M. Pathogenesis and surgical treatment of migraine and neurovascular headaches with rhinogenic trigger. Head Neck. 1992;14(6):467-472.

37. Clerico DM, Fieldman R. Referred headache of rhinogenic origin in the absence of sinusitis. Headache. 1994;34(4):226-229.

38. Clerico DM. Sinus headaches reconsidered: referred cephalgia of rhinologic origin masquerading as refractory primary headaches. Headache. 1995;35(4):185-192.

39. Bektas D, Alioglu Z, Akyol N, Ural A, Bahadir O, Caylan R. Surgical outcomes for rhinogenic contact point headaches. Med Princ Pract. 2011;20(1):29-33. 